\title{
Bloch surface waves in ultrathin waveguides: near-field investigation of mode polarization and propagation
}

\author{
Tristan Sfez, ${ }^{1, *}$ Emiliano Descrovi, ${ }^{2}$ Libo Yu, ${ }^{1}$ Daniele Brunazzo, ${ }^{3}$ Marzia Quaglio, ${ }^{4}$ Lorenzo Dominici, ${ }^{5}$ \\ Wataru Nakagawa, ${ }^{6}$ Francesco Michelotti, ${ }^{5}$ Fabrizio Giorgis, ${ }^{2}$ Olivier J. F. Martin, ${ }^{3}$ and Hans Peter Herzig ${ }^{1}$ \\ ${ }^{1}$ Optics \& Photonics Technology Laboratory, Ecole Polytechnique Fédérale de Lausanne, EPFL-STI-OPT, \\ rue A.-L. Breguet 2, CH-2000 Neuchâtel, Switzerland \\ ${ }^{2}$ Materials and Microsystems Laboratory $\chi$ Lab, Politecnico di Torino, C.so Duca degli Abruzzi 24, \\ 10129 Torino, Italy \\ ${ }^{3}$ Nanophotonics and Metrology Laboratory, Swiss Federal Institute of Technology Lausanne, EPFL-STI-NAM, \\ ELG Station 11, CH-1015 Lausanne, Switzerland \\ ${ }^{4}$ Center for Human Space Robotics of IIT-Italian Institute of Technology, Corso Trento 21, 10129 Torino, Italy \\ ${ }^{5}$ Dipartimento di Energetica, SAPIENZA, Università di Roma and CNISM, via A. Scarpa 16, o0161 Roma, Italy \\ ${ }^{6}$ Electrical and Computer Engineering Department, Montana State University, P.O. Box 173780, Bozeman, \\ Montana 59717-3780, USA \\ *Corresponding author: tristan.sfez@epfl.ch
}

Received March 2, 2010; revised June 1, 2010; accepted June 23, 2010; posted June 29, 2010 (Doc. ID 124950); published July 22, 2010

\begin{abstract}
In this work, we use a multi-heterodyne scanning near-field optical microscope to investigate the polarization and propagation of Bloch surface waves in an ultrathin $(\sim \lambda / 10)$ ridge waveguide. First, we show that the structure sustains three surface modes, and demonstrate selective excitation of each. Then, by numerically processing the experimental data, we retrieve the transverse and longitudinal components of each of the modes, in good agreement with the calculated fields. Finally, we provide an experimental estimation of the effective indices and the dispersion relations of the modes. (C) 2010 Optical Society of America
\end{abstract}

OCIS codes: $240.6690,120.5050,120.5410,180.4243,230.7380,230.4170$.

\section{INTRODUCTION}

Carefully designed periodic dielectric structures may sustain surface electromagnetic states-called Bloch surface waves (BSWs)—within their photonic bandgaps [1]. As surface states, they are bound to the interfaces and may show significant field confinement. Although BSWs in dielectric multilayers have been known since the late 1970's [2], they have recently been reexamined for their potential in sensing applications [3-5].

In a recent paper, the authors demonstrated that an ultrathin $(\sim \lambda / 10)$, low-index, dielectric ridge deposited on a multilayer can act as a waveguide for BSWs [6]. It has also been shown that the lateral confinement of the BSW in the waveguide may be seen as a two-dimensional refractive behavior involving total internal reflection [7]. Although they do not allow subwavelength lateral confinement, the guided BSWs possess several interesting peculiarities. First, their dispersion characteristics are not limited by the properties of a particular material and can be tailored by engineering the multilayer structure. Second, they penetrate further into the outer medium than standard waveguide modes [8]. Third, as these structures consist of dielectric materials, their losses can be made very low. In waveguide-based biosensing (see, e.g., [9]), these structures might provide new opportunities for applications in which the waveguide is made out of functionalized molecular layers of nanometric thick- nesses. Such BSW waveguides are furthermore wavelength scalable and fully compatible with the fabrication technologies for integrated photonic and plasmonic structures.

With the aim of gaining a better understanding of the field distribution in these waveguides, we report on the use of a multi-heterodyne scanning near-field optical microscope (MH-SNOM) [10-14] for the investigation of the modes sustained by the structure. In particular, the polarization characteristics of these modes are investigated. Unlike near-field phase measurements, which are routinely performed nowadays [15-18], polarization measurements in the near field still remain a challenging task $[19,20]$. Although experiments involving the polarization contrast mechanism have been performed for a long time $[21,22]$, measuring the state of polarization itself implies knowledge of the vector field of light, i.e., two orthogonal amplitude components and the relative phase difference between them [23]. Recently, the amplitude components of the field have been measured [24,25]. Measurements involving both the amplitude and the phase of two orthogonal field components have also been carried out [26,27]. In a beautiful experiment, Burresi et al. [28] integrally resolved the state of polarization of the light within a $2 \mathrm{D}$ photonic crystal. However, all these techniques rely on a serial experimental process with control of the polarization in between each measurement. 
Exploiting the capability of the MH-SNOM to simultaneously measure the amplitude and phase of two arbitrary orthogonal components of paraxial (near) fields, we develop a technique for retrieving the field at the sample surface. The method relies on a priori information about the field distribution and on a simple numerical treatment of the experimental data. We apply the technique to the previously mentioned BSW waveguide using a simple energy consideration first tested on the calculated fields.

The paper is organized as follows. After a description of the sample and the numerical method used in this work, we present a detailed description of the MH-SNOM. We demonstrate through a direct near-field visualization that the BSW waveguide sustains three surface modes and that we are able, by tuning the wavelength and choosing the incident polarization, to selectively excite each of them. We then measure the individual transverse and longitudinal field components of each mode, both in amplitude and phase. Finally, their dispersion relations are experimentally deduced.

\section{BSW WAVEGUIDE}

The sample is a hydrogenated amorphous silicon nitride multilayer grown by plasma enhanced chemical vapor deposition (PECVD) on a $500-\mu \mathrm{m}$-thick glass substrate. The refractive index of the layers is tuned by controlling the concentration of ammonia in a $\mathrm{SiH}_{4}+\mathrm{NH}_{3}$ plasma. The multilayer consists of a 10-period stack of high-index $\left(\mathrm{n}_{h}=2.23\right.$ at $\left.\lambda=1530 \mathrm{~nm}\right)$ and low-index $\left(\mathrm{n}_{l}=1.75\right.$ at $\lambda$ $=1530 \mathrm{~nm}$ ) layers whose thicknesses are $\mathrm{d}_{l}=294 \mathrm{~nm}$ and $\mathrm{d}_{h}=240 \mathrm{~nm}$, respectively. The top layer is in contact with the outer medium (air) and has a low index. Details regarding the multilayer fabrication may be found in [12].
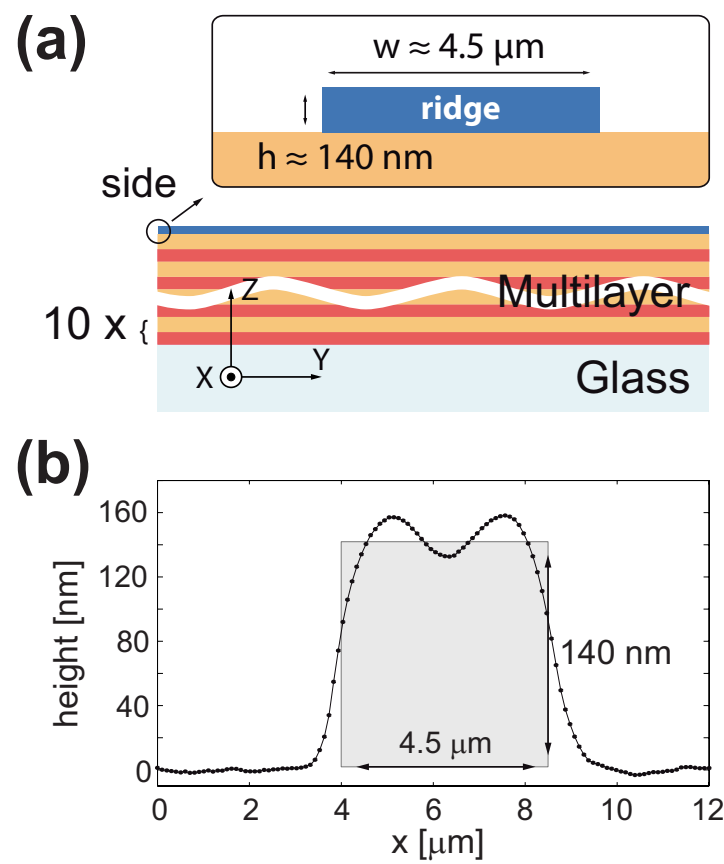

Fig. 1. (Color online) (a) Schematic diagram of the silicon nitride multilayer with a polymeric ridge on top. (b) A topographic cross-section of the ridge as obtained from the shear-force feedback (dotted line). The gray rectangle represents the structure used in the calculation (height $h=140 \mathrm{~nm}$ and width $w=4.5 \mu \mathrm{m}$ ).
Polymeric ridges are then deposited on the top surface. They are fabricated by means of standard photolithographic process after spinning of a positive photoresist (AZ5214E, Clariant $\mathrm{GmbH}, \mathrm{n}_{\text {pol }} \simeq 1.625$ ). Figure 1(a) shows a schematic drawing of the structures. A topographical cross-section measured with the shear-force mechanism (see Section 4) is displayed in Fig. 1(b) (dotted curve). The profile presents two bumps whose heights are approximately $155 \mathrm{~nm}$ with a groove at $130 \mathrm{~nm}$ in between. The mean width of the ridge is approximately $4.5 \mu \mathrm{m}$.

\section{NUMERICAL METHOD}

The spatial distribution of the modes sustained by the BSW waveguide are calculated with a commercial finiteelement package (COMSOL Multyphysics v.3.5a). The calculation domain is enclosed in a perfectly matched layer (PML) to avoid reflections at the boundaries. A convergence analysis is conducted to ensure that the mode effective indices vary by less than $1 \%$. It corresponds to a square computational domain with edges longer than $10 \lambda$, both in the $x$ and $z$ directions (see Fig. 2). The thickness of the PML is chosen to be equal to $1.5 \lambda$. The effective index of the BSW sustained by the bare multilayer is extracted from [12] using the formula $n_{\text {eff }}=\lambda /(2 \pi) \cdot \beta$ and used as an initial guess for rapid convergence. We approximate the waveguide profile by a ridge whose width and height are $w=4.5 \mu \mathrm{m}$ and $h=140 \mathrm{~nm}$, respectively, as indicated by the gray rectangle in Fig. 1(b). The refractive index used in the calculations is $\mathrm{n}_{p o l}=1.625$.

The calculations performed at $\lambda=1520 \mathrm{~nm}$ show that the structure sustains three modes confined at the outer interface of the guide (i.e., surface modes) and laterally confined by the polymeric ridge. Their transverse intensity distributions are shown in Figs. 2(a)-2(c). The polarizations of the modes are parallel to the multilayer interfaces [plane $(x, y)$ in Fig. 1(a)] with a dominant transverse component in the $x$ direction. The fundamental, first- and second-order modes will be referred to as mode 0 , mode 1 , and mode 2 , respectively. The calculations show modes 0 and 2 to be symmetric and mode 1 to be antisymmetric. In Fig. 2(a), mode 0 shows one lobe well confined in the ridge. In Fig. 2(b), mode 1 shows two lobes that are still confined in the ridge but whose lateral evanescent tails penetrate slightly further into the air surrounding the guide. Finally in Fig. 2(c), mode 2 has three lobes with a greater penetration into the outer medium (air).

A detailed description involving the vectorial components of the calculated modes is presented in Section 6 in comparison with experimental measurements.

\section{MULTI-HETERODYNE SCANNING NEAR- FIELD OPTICAL MICROSCOPE}

Optical measurements are performed using a MH-SNOM (see [10-12] and references therein), which is a modified version of a classical heterodyne SNOM. In standard heterodyne SNOM [16], the sample and the probe are placed into one arm-the object arm-of a Mach-Zehnder interferometer. Both the reference and the object arms are frequency shifted by means of acousto-optic modulators 

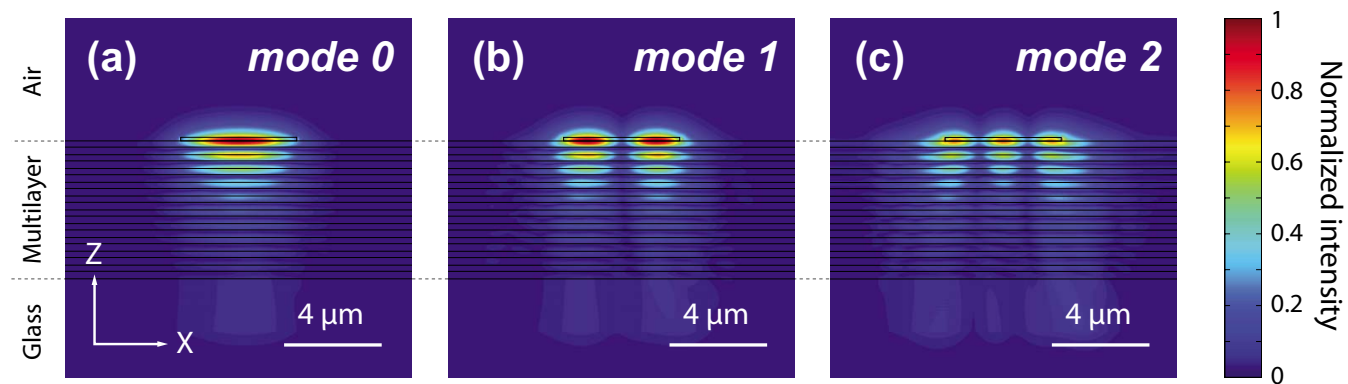

Fig. 2. (Color online) Calculated transverse intensity distributions of the (a) fundamental, (b) first-, and (c) second-order modes ( $\lambda$ $=1520 \mathrm{~nm}$ ).

(AOMs). Finally, the reference and object signal are superimposed and sent to a detector. The frequency difference $\Delta f$ generates a beat signal whose intensity at the detector is

$$
I(t)=A_{r}^{2}+A_{o}^{2}+2 A_{r} A_{o} \cos \left[2 \pi \Delta f \cdot t+\left(\varphi_{o}-\varphi_{r}\right)\right]
$$

where $A_{r}$ and $A_{o}$ are the amplitude of the reference and object channels, respectively, and $\varphi_{r}$ and $\varphi_{o}$ are their relative phases. This signal is sent to a lock-in amplifier and compared to an electronic reference at frequency $\Delta f$. The outputs amplitude $R$ and phase $\Phi$ of the lock-in amplifier are

$$
R=2 A_{r} A_{o} \quad \text { and } \quad \Phi=\varphi_{o}-\varphi_{r} .
$$

Since the reference amplitude and phase are constant, $R$ is proportional to the field amplitude at the surface of the sample and $\Phi$ corresponds to its optical phase.

In a multi-heterodyne SNOM, both the reference and the object arms are split again into two orthogonally polarized beams, and each of the four channels is shifted by a different frequency [10].

A schematic diagram of the optical setup constituting the MH-SNOM is presented in Fig. 3. Except for the sample illumination zone, all optical paths are fibered. Blue segments correspond to polarization-maintaining fibers (PMF) and yellow ones to single-mode fiber (SMF). PMFs can be oriented to rotate the polarization of the beams in any arm whenever needed. The fibered output of the laser source (Agilent 81682A, spectral range
$1460-1580 \mathrm{~nm})$ is sent to a polarization beam splitter (PBS) through a polarization controller. The power ratio between the two outputs of the PBS is adjusted by acting on the polarization controller. The two outputs of the PBS initiate the reference and object arms. Each of the two arms is again split using a 50/50 nonpolarizing beam splitter (BS). The four channels are then individually frequency shifted using AOMs driven at different frequencies. The object channels $o 1$ and $o 2$ are then recombined with a PBS in a PMF, this fiber being rotated to generate TE and TM illuminating beams. The two references $r 1$ and $r 2$ are recombined in a SMF with a PBS. Their orthogonality, as well as their linearity, is well preserved up to the detector. Thus, they constitute a basis $\left(\hat{\mathbf{x}}^{\prime}, \hat{\mathbf{y}}^{\prime}\right)$ on which are projected the object beams. This basis is a priori not aligned with the one $(\hat{\mathbf{x}}, \hat{\mathbf{y}})$ at the sample surface.

At the surface of the sample, the light is collected with a SNOM probe (Lovalite, aperture $200 \mathrm{~nm}, 70 \mathrm{~nm} \mathrm{Al}$ coated, SMF) and superimposed on the references using a 99/1 BS. At the detector, the signal is therefore a superposition of four beams of different optical frequencies: six beat signals are hence generated. Among them, the four beat signals corresponding to a particular reference with a particular object channel are sent to four lock-in amplifiers.

Equation (2) independently holds for each of the four beat signals, and the four lock-ins' output amplitudes $R_{r 1, r 2}^{T E, T M}$ and phases $\Phi_{r 1, r 2}^{T E, T M}$ are

$$
R_{r 1, r 2}^{T E, T M}=2 A_{r 1, r 2} A_{x y^{\prime}}^{T E, T M}
$$

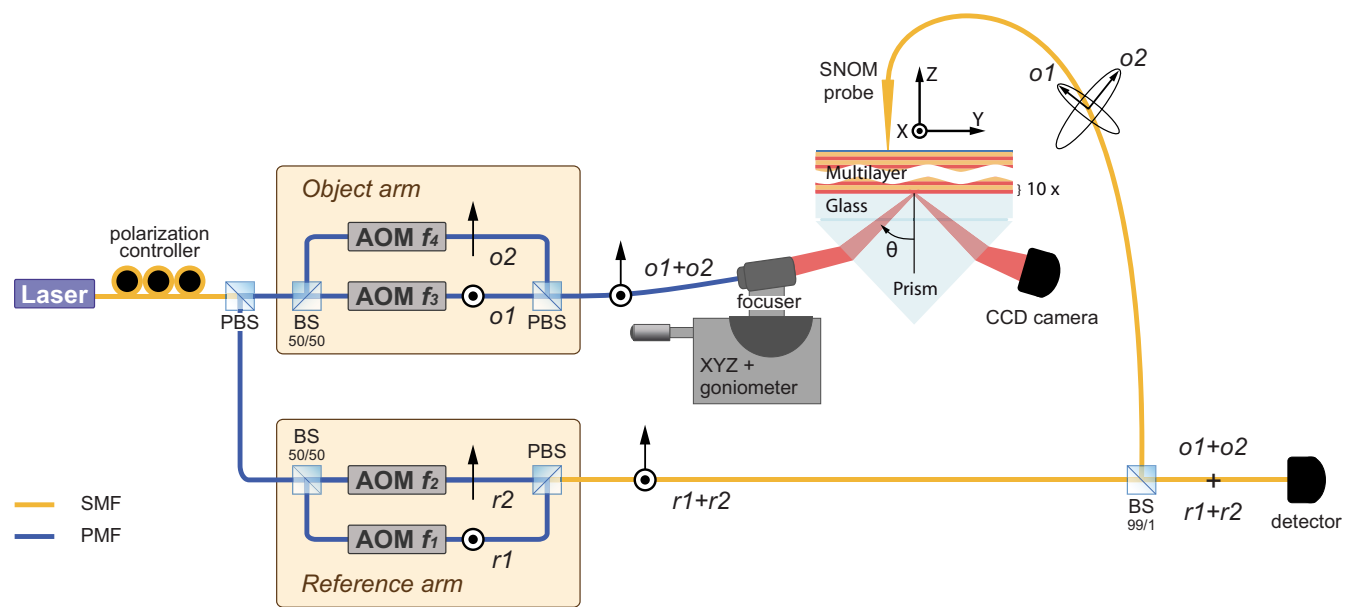

Fig. 3. (Color online) Schematic diagram of the multi-heterodyne interferometer constituting the MH-SNOM. The sample is illuminated in the Kretschmann configuration. 


$$
\Phi_{r 1, r 2}^{T E, T M}=\varphi_{x y^{\prime}}^{T E, T M}-\varphi_{r 1, r 2}
$$

with $A_{r 1}, \varphi_{r 1}, A_{r 2}$, and $\varphi_{r 2}$ being respectively the amplitudes and phases of the linear references $r 1$ and $r 2$, and $A_{x y^{\prime}}^{T E}, \varphi_{x y^{\prime}}^{T E}, A_{x y^{\prime}}^{T M}, \varphi_{x y^{\prime}}^{T M^{\prime}}$ being the amplitudes and phases of the object channels $o 1$ and $o 2$ in the detector frame of reference $\left(\hat{\mathbf{x}}^{\prime}, \hat{\mathbf{y}}^{\prime}\right)$. The lock-in amplitudes and phases are acquired at each point $(x, y)$ of the raster scanned map. We stress that, in our experiment, $A_{r 1} \simeq A_{r 2}$; the four amplitudes $R_{r 1, r 2}^{T E, T M}$ can therefore be compared quantitatively.

The sample is illuminated with a snap-on focuser (divergence $\delta \simeq 4^{\circ}$ ) producing a spot size of approximately $20 \mu \mathrm{m}$ at the top interface of the sample. The focuser is mounted on a goniometric stage allowing the adjustment of the angle of incidence $\theta$. The sample is illuminated in the Kretschmann configuration using a BK7 prism $\left(\mathrm{n}_{p}\right.$ $=1.50$ ) optically connected to the sample with an indexmatching liquid. The reflected light from the prism is monitored with an IR-sensitive CCD camera in a classical M-line configuration [29]. The probe is maintained in close proximity to the sample by means of a shear-force feedback system (SNOM Control Unit, APE Research), which also provides topographic information about the sample.

Since the beams are labeled with different beat frequencies, it is therefore possible to simultaneously map the evanescent fields generated by the two TE- and TMpolarized illuminating beams. It is worth noting that the detected near fields may no longer correspond to such polarizations. For example, the allowed states of polarization in a waveguide are imposed by the waveguide modes (i.e., by the materials and geometries); so if a nonzero signal is detected while scanning the waveguide, it means that a fraction of the input light has been coupled into the waveguide, and the polarization that was initially TE (resp. TM) now has the polarization of the waveguide modes. In the following work, TE and TM polarizations refer to the measured fields that are generated by these incident polarizations.

\section{SELECTIVE EXCITATION OF THE MODES}

At first we set the angle of incidence to $\theta=56^{\circ}$ at $\lambda$ $=1538 \mathrm{~nm}$. Figures 4(a) and 4(b) respectively show the intensity distributions corresponding to TE and TM illumination, as simultaneously obtained with the MH-SNOM. The Airy patterns seen in both maps indicate the region of incidence, i.e., the zone where the focused incident light strikes the sample. Both patterns demonstrate guidance within the polymeric ridge over more than $350 \mu \mathrm{m}$, although weaker for the TM case. Up to $200 \mu \mathrm{m}$, the TE distribution looks like the fundamental transverse mode of a classical dielectric ridge waveguide. The presence of a topographical defect (inset) at $y \simeq 200 \mu \mathrm{m}$ considerably affects the spatial distribution of the field propagating beyond this point, although it is still guided. This behavior experimentally demonstrates the multimodal structure of the waveguide: due to the defect, the energy which was mainly in the fundamental mode is redistributed among the available modes. The pattern is asymmetric and sug-

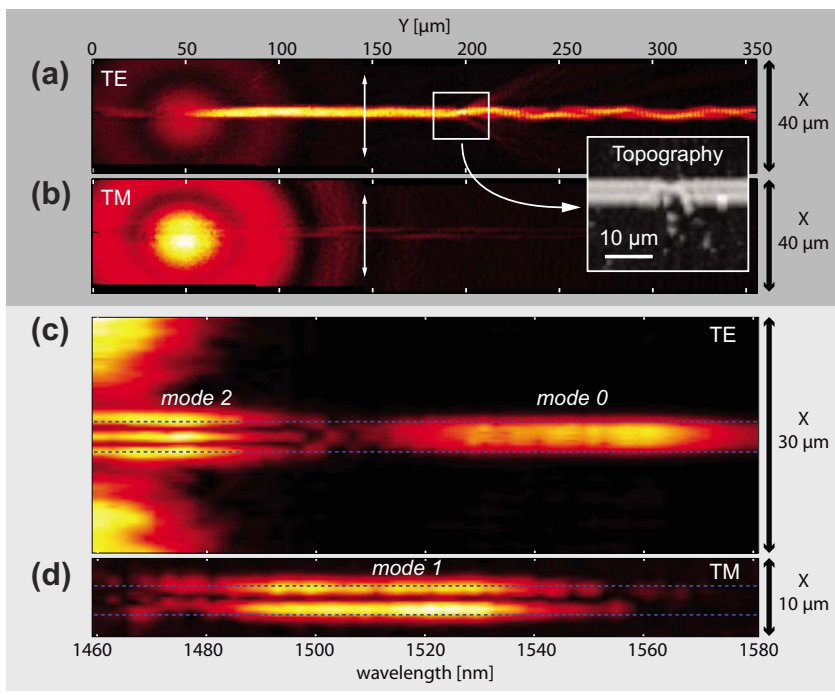

Fig. 4. (Color online) In (a) and (b), near-field intensities over the waveguide under TE- and TM-polarized illumination, respectively $(\lambda=1538 \mathrm{~nm})$. The Airy-type patterns at left indicate the region where the incident light impinges on the sample surface. The inset shows the topographical defect responsible for the change in the propagating optical mode. (c) and (d) present $(\lambda, x)$ plots for TE and TM polarization, respectively. The $x$ line follows the white arrows displayed in (a) and (b).

gests a beating between symmetric and asymmetric modes [32]. This remark also holds true for the TM distribution.

Due to their dispersion, at a fixed angle of incidence $\theta$, the transverse modes are expected to be excited at different wavelengths. In order demonstrate this effect and to characterize the modes, we set the SNOM tip in a fixed $y$ position (the white line shown in Figs. 4(a) and 4(b)) and scan a line across the waveguide along the $x$ direction. The $y$ position is chosen so as to lie outside the coupling region and to monitor the unperturbed surface modes of the structure. We then perform $x$ scans while sweeping the wavelength in the interval between $1460 \mathrm{~nm}$ and $1580 \mathrm{~nm}$.

The measurements are shown in Figs. 4(c) and 4(d) for the TE and TM polarizations, respectively. The scanning length is $30 \mu \mathrm{m}$ (only $10 \mu \mathrm{m}$ are displayed on the TM map). The ridge edges are marked with the dashed lines. Starting at longer wavelengths (1515-1580 nm), the TE map shows a one-lobe elongated spot centered on the ridge. Then the TM map reveals a two-lobe elongated spot at intermediate wavelengths $(1480-1540 \mathrm{~nm})$. Finally at shorter wavelengths (1460-1490 nm), the TE pattern shows a three-lobe spot inside the ridge. Since the scanning zone is approximately $100 \mu \mathrm{m}$ away from the incident region, these three spots are identified as mode 0 (one lobe), mode 1 (two lobes) and mode 2 (three lobes).

The intensity pattern of mode 2 is surrounded by light corresponding to the BSW in the bare multilayer $\left(\mathrm{BSW}_{\text {bare }}\right)[12]$. The dispersion curve of the $\mathrm{BSW}_{\text {bare }}$ therefore partially overlaps the dispersion curve of mode 2 . No higher-order modes are hence allowed within the waveguide, which is consistent with the calculations. Fortunately, from the incident region and up to the scanning zone, the $\mathrm{BSW}_{\text {bare }}$ propagates slightly away from the waveguide, making possible independent measurements 
on mode 2. Also, the mode spatial extent increases with the mode order: $3.8 \mu \mathrm{m}, 4.7 \mu \mathrm{m}$, and $6.1 \mu \mathrm{m}$ (FWHM) for modes 0,1 , and 2 , respectively.

Due to its antisymmetry, it is expected that TEpolarized light cannot excite mode 1 . However, it may seem surprising that TM-polarized light couples to this mode, accounting for its in-plane polarization. A rigorous survey of the coupling mechanism should, in principle, involve an overlap integral. We have performed additional calculations (not shown here) using a rigorous coupledwave analysis (RCWA). It is indeed seen that only a TMpolarized plane wave can excite mode 1 .

By choosing the wavelength and the excitation polarization, we can therefore selectively excite the three modes sustained by the structure.

\section{VECTORIAL COMPONENTS OF THE MODES}

\section{A. Method}

In our experiment, we must distinguish two distinct frames of reference. At the detector plane, the reference frame $\left(\hat{\mathbf{x}}^{\prime}, \hat{\mathbf{y}}^{\prime}\right)$ is fixed by the two linearly polarized references of the multi-heterodyne interferometer. At the sample surface, the reference frame $(\hat{\mathbf{x}}, \hat{\mathbf{y}})$ is set according to the BSW waveguide. This situation is conceptually depicted in Fig. 5. The function that relates the expression of the electromagnetic field in these two bases is hereafter referred to as the polarization transfer matrix (PTM).

The existence of such a PTM has already been demonstrated for a dielectric probe and for a paraxial (perpendicular to the probe axis) and linear input state of polarization (SOP) [10]. In our experiment, the calculations show the electric field $\mathbf{E}$ of the modes 0,1 , and 2 to be also paraxial: $\mathbf{E}$ is parallel to the multilayer interfaces and dominantly transverse $\left(\mathrm{E}_{x}\right)$. E possesses, however, a small longitudinal component $\mathrm{E}_{y}$ in the propagation direction $y$. The goal is hence to find $\mathrm{E}_{x}$ and $\mathrm{E}_{y}$.

As we are interested in the vectorial structure of the field, we compute the measured complex TE and TM vec-

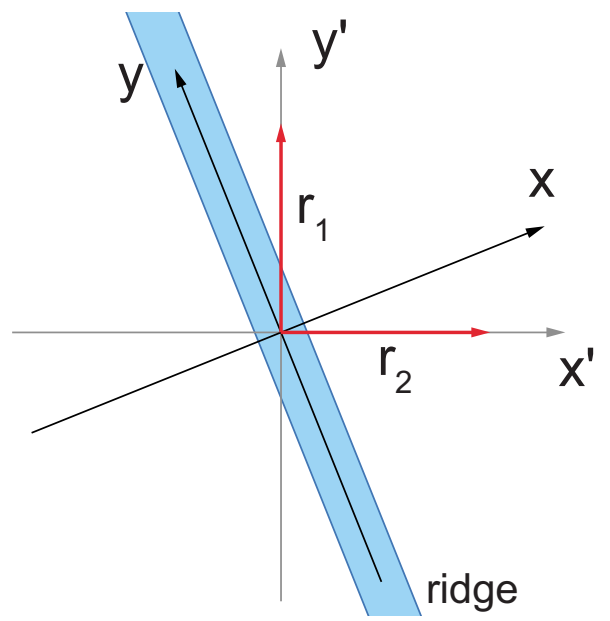

Fig. 5. (Color online) At the detector plane, the reference frame $\left(\hat{\mathbf{x}}^{\prime}, \hat{\mathbf{y}}^{\prime}\right)$ is fixed by the two linearly polarized references of the multi-heterodyne interferometer. At the sample surface, the reference frame $(\hat{\mathbf{x}}, \hat{\mathbf{y}})$ is set according to the BSW waveguide. tor fields, $\mathbf{E}_{\text {det }}^{T E}$ and $\mathbf{E}_{\text {det }}^{T M}$, in $\left(\hat{\mathbf{x}}^{\prime}, \hat{\mathbf{y}}^{\prime}\right)$. With the experimental amplitudes and phases of Eqs. (3), these fields become

$$
\begin{aligned}
& \mathbf{E}_{d e t}^{T E}(x, y)=\left(\begin{array}{l}
R_{r 1}^{T E}(x, y) \cdot \exp \left[i \cdot \Phi_{r 1}^{T E}(x, y)\right] \\
R_{r 2}^{T E}(x, y) \cdot \exp \left[i \cdot \Phi_{r 2}^{T E}(x, y)\right]
\end{array}\right), \\
& \mathbf{E}_{d e t}^{T M}(x, y)=\left(\begin{array}{l}
R_{r 1}^{T M}(x, y) \cdot \exp \left[i \cdot \Phi_{r 1}^{T M}(x, y)\right] \\
R_{r 2}^{T M}(x, y) \cdot \exp \left[i \cdot \Phi_{r 2}^{T M}(x, y)\right]
\end{array}\right) .
\end{aligned}
$$

The $(x, y)$ dependence states that the fields are measured at each position of the probe. The difficulty in linking the field at the detector plane to that at the sample surface arises because we don't know a priori the relative orientation at the detection plane of the TE field (resp. TM) with respect to the basis. Moreover, due to some birefringence that may occur within the probe [10] and in the optical path from the probe to the detector, the initial SOPS of the modes 0,1 , and 2 may reach the detector slightly altered.

Since any SOP can be reached with a proper combination of a quarter-wave and a half-wave plate, $\mathbf{J}_{4}$ and $\mathbf{J}_{2}$, respectively, the field at the sample surface $\mathbf{E}_{\text {sample }}^{T E, T M}$ may be expressed as

$$
\mathbf{E}_{\text {sample }}^{T E, T M}(x, y)=\mathbf{M} \cdot \mathbf{E}_{\text {det }}^{T E, T M}(x, y),
$$

where $\mathbf{M}=\mathbf{R}\left(-\alpha_{1}\right) \mathbf{J}_{4} \mathbf{R}\left(\alpha_{1}\right) \cdot \mathbf{R}\left(-\alpha_{2}\right) \mathbf{J}_{2} \mathbf{R}\left(\alpha_{2}\right)$ is the PTM and $\mathbf{R}$ is the coordinate transform matrix [33]. The PTM is therefore modeled as an equivalent birefringent Jones matrix. The above equation represents the inverse problem of finding the field at the sample surface from the field at the detector. The physical propagation would indeed be represented by $\mathbf{E}_{\text {det }}^{T E, T M}=\mathbf{M}^{-1} \cdot \mathbf{E}_{\text {sample }}^{T E, T M}$. The issue is now to numerically find the angles $\alpha_{1}$ and $\alpha_{2}$ that lead to a separation of $\mathrm{E}_{x}$ and $\mathrm{E}_{y}$.

To do so, an additional criterion is required. For any given values of $\left(\alpha_{1}, \alpha_{2}\right)$, the rule should indicate the degree to which $\mathbf{E}_{\text {sample }}^{T E, T M}(x, y)$ calculated with Eq. (5) matches the expected maps. Since the calculations show $E_{y}$ to be small compared to $E_{x}$ (ratio $\sim 1: 5$ ), the criterion is to find the best pair $\left(\alpha_{1}, \alpha_{2}\right)$ that minimize the intensity of one component of $\mathbf{E}_{\text {sample }}^{\text {TE,TM }}(x, y)$ while maximizing the other. We therefore seek for the best matrix $\mathbf{M}$ that, applied pointby-point to $\mathbf{E}_{\text {det }}^{T E, T M}$, minimizes the intensity of one component over the entire map (integrated intensity over the entire map). This method has been first tested with the calculated fields. Starting from a projection of the field on two random and linear basis elements, $\mathrm{E}_{x}$ and $\mathrm{E}_{y}$ have been successfully retrieved.

The equivalent hardware procedure to the previous method consists of introducing a polarization controller after the probe. Consequently, $\mathrm{E}_{x}$ and $\mathrm{E}_{y}$ could be physically aligned with the reference beams $r 1$ and $r 2$, and therefore measured. However, the experimenter also needs a priori knowledge of the expected field for assessing the accuracy of the polarization controller alignment for each measurement. The experimental procedure therefore involves an alternation of measurements and polarization controller transformations. Since SNOM imaging is a scanning process, the collection of the individual SNOM images would be extremely time consuming. 


\section{B. Field Distributions of the Modes}

We apply step by step the numerical method developed in the previous section. As demonstrated in Section 5, by tuning the wavelength and choosing the excitation polarization, we can selectively excite each of the three modes sustained by the waveguide. The modes 0 and 2 are excited with TE-polarized light at $\lambda=1568 \mathrm{~nm}$ and $\lambda$ $=1478 \mathrm{~nm}$, respectively. The mode 1 is excited with TMpolarized light at $\lambda=1514 \mathrm{~nm}$. The sub-figures (A-C) of Fig. 6 respectively show the results for modes 0,1 , and 2 .

Within each sub-figure, $(a-b)$ and $(c-d)$ show the raw amplitudes and phases resulting from the interference of the object beam with the references $r 1$ and $r 2$. We arbitrarily choose to minimize the field intensity corresponding to the component $(\mathrm{c}-\mathrm{d})$. The processed amplitude and the phase of this component are shown in $(\mathrm{g}-\mathrm{h})$, respectively. The component $(a-b)$ is therefore automatically maximized and displayed in (e-f). In order to compare the measurements with the calculations, we compute, from the amplitudes and phases shown in $(\mathrm{e}-\mathrm{h})$, the real parts of $E_{x}$ and $E_{y}$. (i-j) and $(\mathrm{k}-\mathrm{l})$ respectively show the measured and calculated real parts of $E_{x}$ and $E_{y}$. The calculated maps are obtained as follows. From the computed transverse field distributions, we extract a line at $7 \mathrm{~nm}$ above the ridge interface. Then, we make it propagate with the effective index obtained from the same simulation (mode 0: $n_{\text {eff }}=1.256$ at $\lambda=1568 \mathrm{~nm}$, mode 1: $n_{\text {eff }}$ $=1.274$ at $\lambda=1514 \mathrm{~nm}$, mode $2: n_{\text {eff }}=1.273$ at $\lambda=1478 \mathrm{~nm}$ ). For the sake of clarity, a global phase delay is added to the calculated real parts. The gray lines represent the edges of the ridge as provided by the shear-force topography.

For each of the modes, the measured real parts of the transverse and longitudinal components are found in good agreement with the calculated ones. First, their spatial distributions have almost the same extent. Second, the amplitude ratios $\left|E_{x}\right| /\left|E_{y}\right|$ of the measured and simulated values are satisfactorily close: 5 versus 7.5 for mode 0 ; 3.8 versus 4 for mode 1 ; and 3.6 versus 2.9 for mode 2 . Third, the symmetry of each measured component is retrieved. Because $E_{x}$ is stronger-and so defines the mode symmetry-we verify that modes 0 and 2 are symmetric whereas mode 1 is antisymmetric.

Comparing the results of the three modes, we can draw two overall conclusions. First, the measured and calculated modes shows slightly different periodicities. This may be due either to a waveguide cross-section that departs from the ideal rectangular shape of the simulated structure [see Fig. 1(b)], or to a possible deviation between the real and theoretical effective indices of the polymer. Furthermore, the sample has a slightly inhomogeneous topography leading to some local fluctuations of the effective indices.

Second, in this particular experiment, the relative phase difference between $E_{x}$ and $E_{y}$ is not measured. The apparent relative phase differences appearing in Fig. 6 $(\mathrm{A}-\mathrm{C})$ (i) and (k) arise because of the arbitrary optical path differences within the multi-heterodyne interferometer. This can be solved in the future by a proper calibration of the system using a field of known polarization or by implementing a two-detector detection system such as in $[10,11]$.

\section{DISPERSION RELATIONS OF THE MODES}

From the amplitude and phase of an optical signal, one can compute the complex field [30]. Considering lines in the $y$ direction (propagation direction), the four amplitudes and phases of Eqs. (3) lead to the four scalar complex fields

$$
V_{r 1, r 2}^{T E, T M}(y)=R_{r 1, r 2}^{T E, T M}(y) \cdot \exp \left[i \Phi_{r 1, r 2}^{T E, T M}(y)\right]
$$

A Fourier analysis of these complex fields provides the $k_{y}$ components of the in-plane wavevectors of the optical field. For guided or bound modes, the propagation constants can be deduced $[12,14,17,31]$ and the effective indices determined through the relation $n_{\text {eff }}=\lambda k_{y} /(2 \pi)$. As the resolution in the Fourier space is inversely proportional to the scanning length $L$, we use the entire travel range of the piezo actuator $(L=100 \mu \mathrm{m})$. This condition leads to a theoretical wavevector resolution of $d k_{y}$ $=2 \pi / L=6.3 \cdot 10^{4} \mathrm{~m}^{-1}$, which in these experiments corresponds to a low-effective-index resolution of $d n_{\text {eff }}$ $=\lambda d k_{y} /(2 \pi) \simeq 0.015$. This poor resolution will be overcome by a statistical treatment of the data acquired at different wavelengths.

At a given wavelength, because the transverse field distributions of the modes are different, six high-resolution $y$ lines are acquired on both sides of the ridge. For each line, the FFT spectra are computed on the four complex fields of Eqs. (6). The relevant peaks are identified and their effective indices calculated. Two representative TE and TM spectra are shown in Fig. 7(a) $(\lambda=1530 \mathrm{~nm})$. At this wavelength, the analysis performed on Fig. 4(c) and 4(d) allows identification of the dominant TE and TM peaks with modes 0 and 1 , respectively. These peaks are only separated by $d k_{y}$, which highlights the low-effective-index resolution.

This procedure is repeated over the entire wavelength range $(1460-1580 \mathrm{~nm})$ in steps of $6 \mathrm{~nm}$. The measured effective indices are indicated by the filled circles in Fig. 7(b). The diameters account for the peak occurrences: six scanning lines with four complex fields leads to 24 spectra per wavelength. Three regions can be distinguished: one at larger wavelengths (1520-1580 nm) and higher $n_{\text {eff }}$, an intermediate zone (1484-1552 nm) with intermediate $n_{\text {eff, }}$ and a region at shorter wavelengths (1460-1502 nm) and lower $n_{\text {eff. }}$ The wavelength ranges covered by the different modes [see Fig. 4(c) and 4(d)] and the fact that lower-order modes have higher $n_{\text {eff }}$ identify these regions with modes 0,1 , and 2 , respectively. Linear curve fits accounting for the weights (occurrences) of the experimental values are plotted with the dotted curves (blue online).

In Fig. 7(c), the fitted curves are reported in a dispersion diagram. The dispersion curves of modes 0,1 , and 2 are, respectively, displayed in red, green, and blue. In addition, the measured dispersion curves of the bare multilayer as obtained from far-field measurements [6], and the calculated dispersion curve of the multilayer coated with a $140-\mathrm{nm}$-thick polymeric layer $\left(\mathrm{n}_{\text {pol }}=1.625\right)$ are reported. As expected, the dispersion curves of the modes lie between the bare and coated ones. An increase in the mode order decreases the field fraction confined in 
(A) Mode 0

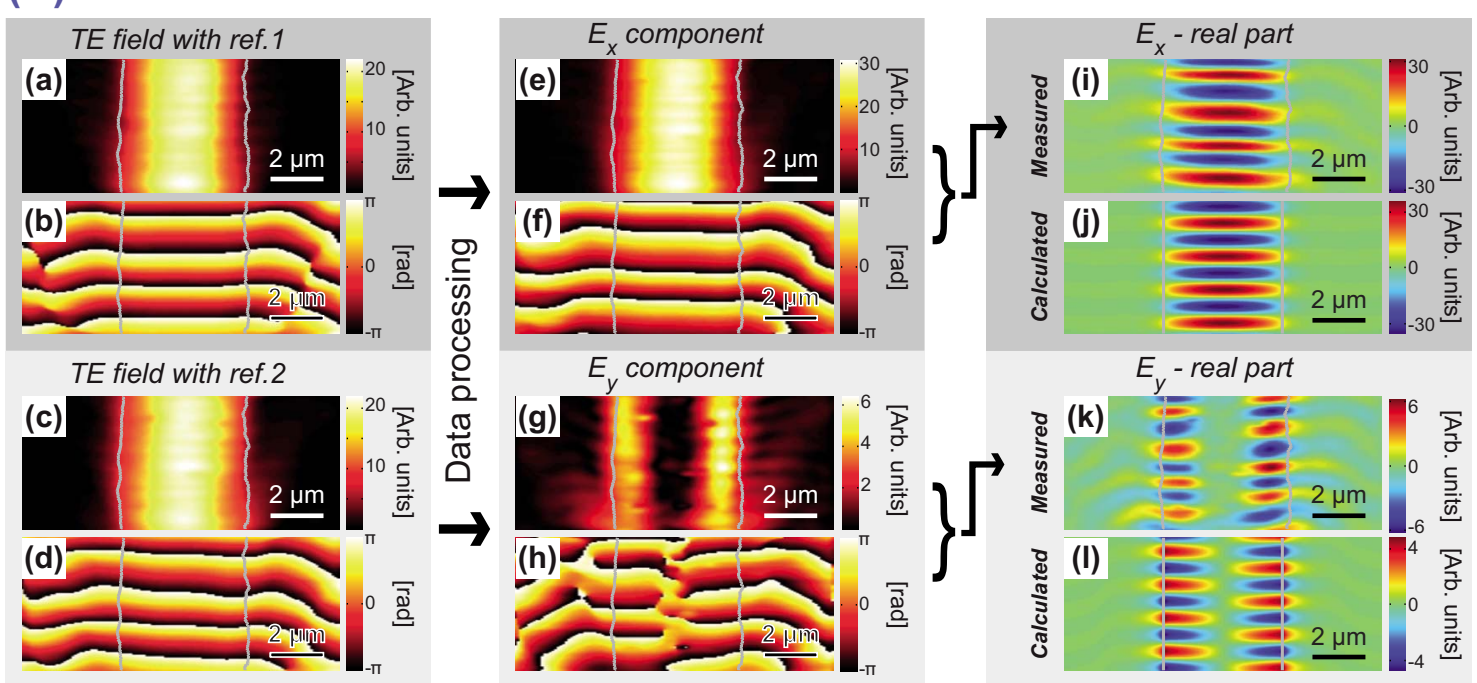

(B) Mode 1

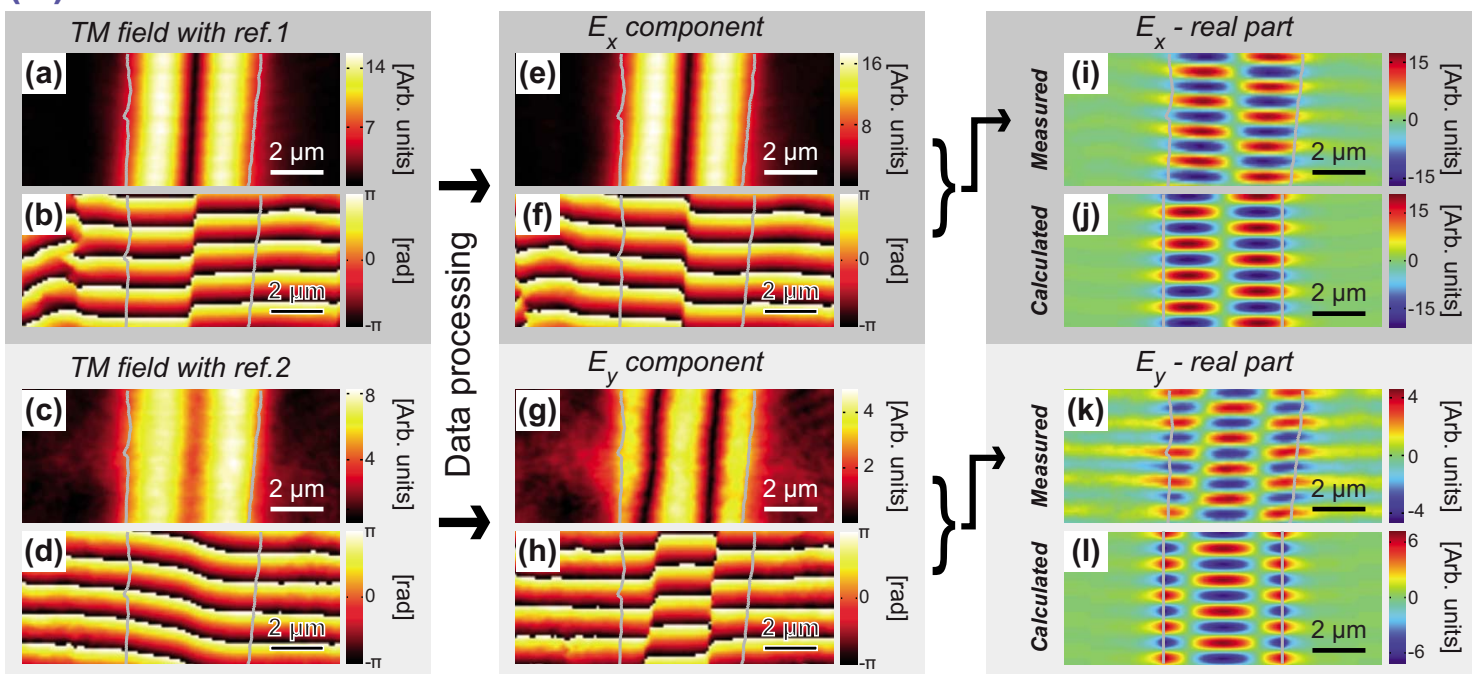

(C) Mode 2

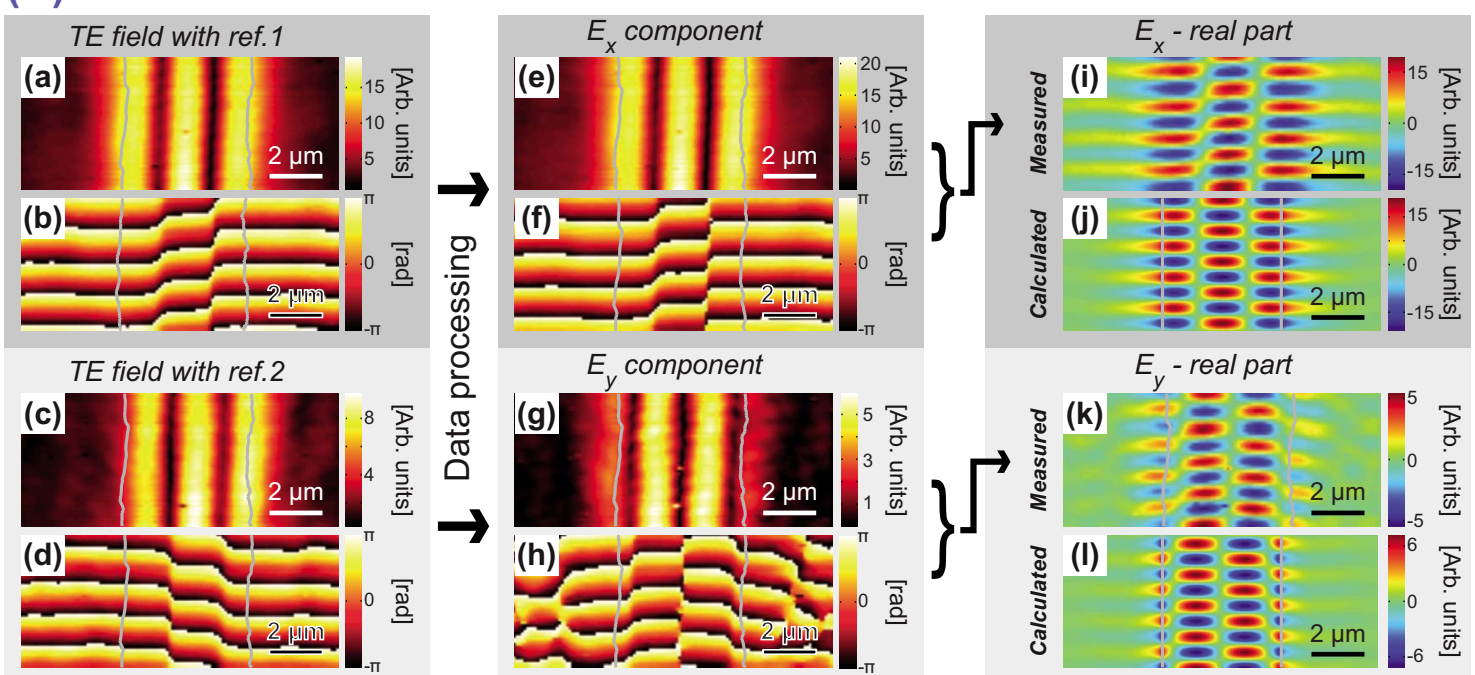

Fig. 6. (Color online) (A): (a-b) and (c-d), the amplitudes and phases of the mode 0 projected onto the two reference beams $r 1$ and $r 2$. In $(\mathrm{e}-\mathrm{f})$ and $(\mathrm{g}-\mathrm{h})$, the respective amplitudes and phases of $E_{x}$ and $E_{y}$ retrieved after numerical data processing. In (i-j) and (k-l), the measured and calculated real parts of $E_{x}$ and $E_{y}(\lambda=1568 \mathrm{~nm})$. In (B) and (C), the equivalent for mode $1(\lambda=1514 \mathrm{~nm})$ and mode 2 $(\lambda=1478 \mathrm{~nm})$. 

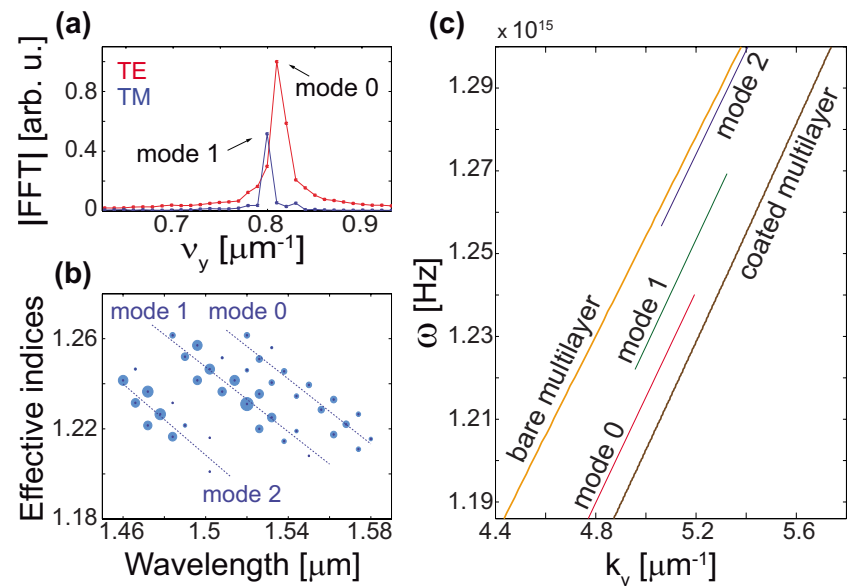

Fig. 7. (Color online) (a) Typical Fourier spectrum obtained for both TE- and TM-polarized fields along the BSW propagation direction $(\lambda=1530 \mathrm{~nm})$. (b) Repartition of the measured effective indices over the whole wavelength range. The filled circle diameters account for the peak occurrences. The dotted lines are the linear curve fits. (c) The corresponding dispersion relations of the modes. In addition the dispersion relations of the bare multilayer and coated multilayer (with a 140 -nm-thick polymeric layer) are also reported.

the ridge region, and the mode effective index therefore decreases, leading to the observed blueshifts of the higher-order modes.

Despite of the low resolution in terms of the effective indices, this experiment shows that accounting for the tunability of the system, the individual dispersion curves of the three modes sustained by the BSW waveguide can be measured.

\section{CONCLUSION}

A multi-heterodyne scanning near-field optical microscope (MH-SNOM) has been used to investigate the propagation and polarization properties of BSW modes in an ultrathin $(\sim \lambda / 10)$ polymeric ridge waveguide. We have experimentally demonstrated that the BSW waveguide sustains three modes and that we were experimentally able to selectively excite them by tuning the wavelength and choosing the excitation polarization. The individual dispersion relations of the modes have been measured and we have shown that they lie, as expected, between the dispersion curves of the bare and coated (140-nmthick polymeric film) multilayer. Exploiting the ability of the MH-SNOM to simultaneously detect in amplitude and phase two arbitrary orthogonal components of the near field, we were able to solve the inverse problem of retrieving the field components of the modes at the sample surface from the fields at the detector plane. This task has been achieved through a simple a priori energy consideration with the calculations indeed showing the longitudinal field component to be weaker than the transverse one. The individual transverse and longitudinal components of each of the modes have hence been measured, and a good agreement with the simulation results was found. By adapting the optimization criterion, this method can be applied to a broad variety of near-field phenomena including waveguiding structures, cavities, resonators, etc. To a certain extent, we can even think of applying this tech- nique without any preliminary information on the desired field but by analyzing the data with mathematical tools such as symmetries. This work provides new insights on the propagation of guided BSWs on ultrathin guiding structures that might be fruitfully exploited in a variety of applications in the domain of low-dimensional optics.

\section{ACKNOWLEDGMENTS}

This work is supported by the Swiss National Science Foundation, by the Piedmont regional project CIPE 2008 "Photonic biOsensors for Early caNcer dIagnostiCS (PHOENICS)" and the Science and Technology Atheneum Research Programme of the SAPIENZA University. The authors also thank Dr. C. Ricciardi for the multilayer deposition.

\section{REFERENCES}

1. R. D. Meade, K. D. Brommer, A. M. Rappe, and J. D. Joannopoulos, "Electromagnetic Bloch waves at the surface of a photonic crystal," Phys. Rev. B 44, 10961-10964 (1991).

2. P. Yeh, A. Yariv, and A. Y. Cho, "Optical surface waves in periodic layered media," Appl. Phys. Lett. 32, 104-105 (1978).

3. F. Villa, L. E. Regalado, F. Ramos-Mendieta, J. GasparArmenta, and T. Lopez-Ríos, "Photonic crystal sensor based on surface waves for thin-film characterization," Opt. Lett. 27, 646-648 (2002).

4. M. Liscidini and J. E. Sipe, "Enhancement of diffraction for biosensing applications via Bloch surface waves," Appl. Phys. Lett. 91, 253125 (2007).

5. E. Descrovi, F. Frascella, B. Sciacca, F. Geobaldo, L. Dominici, and F. Michelotti, "Coupling of surface waves in highly defined one-dimensional porous silicon photonic crystals for gas sensing applications," Appl. Phys. Lett. 91, 241109 (2007).

6. E. Descrovi, M. Quaglio, T. Sfez, D. Brunazzo, L. Dominici, F. Michelotti, H. P. Herzig, O. J. F. Martin, and F. Giorgis, "Guided Bloch surface waves on ultra-thin polymeric ridges," Nano Lett. 10, 2087-2091 (2010).

7. T. Sfez, E. Descrovi, L. Yu, M. Quaglio, L. Dominici, W. Nakagawa, F. Michelotti, F. Giorgis, and H. P. Herzig, "Twodimensional optics on silicon nitride multilayer: refraction of Bloch surface waves," Appl. Phys. Lett. 96, 151101 (2010).

8. V. N. Konopsky and E. V. Alieva, "Photonic crystal surface waves for optical biosensors," Anal. Chem. 79, 4729-4735 (2007).

9. E. Gizeli and C. R. Lowe, Biomolecular Sensors (Taylor \& Francis, 2002).

10. R. Dändliker, P. Tortora, L. Vaccaro, and A. Nesci, "Measuring three-dimensional polarization with scanning optical probes," J. Opt. A, Pure Appl. Opt. 6, S18-S23 (2004).

11. P. Tortora, R. Dändliker, W. Nakagawa, and L. Vaccaro, "Detection of non-paraxial optical fields by optical fiber tip probes," Opt. Commun. 259, 876882 (2006).

12. E. Descrovi, T. Sfez, L. Dominici, W. Nakagawa, F. Michelotti, F. Giorgis, and H. P. Herzig, "Near-field imaging of Bloch surface waves on silicon nitride one-dimensional photonic crystals," Opt. Express 16, 5453-5464 (2008).

13. T. Sfez, E. Descrovi, L. Dominici, W. Nakagawa, F. Michelotti, F. Giorgis, and H. P. Herzig, "Near-field analysis of surface electromagnetic waves in the bandgap region of a polymeric grating written on a one-dimensional photonic crystal," Appl. Phys. Lett. 93, 061108 (2008).

14. B. Bai, X. Meng, J. Laukkanen, T. Sfez, L. Yu, W. Nakagawa, H. P. Herzig, L. Li, and J. Turunen, "Asymmetrical excitation of surface plasmon polaritons on blazed gratings at normal incidence," Phys. Rev. B 80, 035407 (2009).

15. M. L. M. Balistreri, J. P. Korterik, L. Kuipers, and N. F. van 
Hulst, "Local observations of phase singularities in optical fields in waveguide structures," Phys. Rev. B 85, 294-297 (2000).

16. A. Nesci, R. Dändliker, and H. P. Herzig, "Quantitative amplitude and phase measurement by use of a heterodyne scanning near-field optical microscope," Opt. Lett. 26, 208210 (2001).

17. A. Huber, N. Ocelic, D. Kazantsev, and R. Hillenbrand, "Near-field imaging of mid-infrared surface phonon polariton propagation," Appl. Phys. Lett. 87, 081103 (2005).

18. B. Deutsch, R. Hillenbrand, and L. Novotny, "Near-field amplitude and phase recovery using phase-shifting interferometry," Opt. Express 16, 494-501 (2008).

19. J.-J. Greffet and R. Carminati, "Image formation in nearfield optics," Prog. Surf. Sci. 56, 133-237 (1997).

20. S. I. Boshevolnyi, B. Vohnsen, and E. A. Bozhevolnaya, "Transfer functions in collection mode scanning near-field optical microscopy," Opt. Commun. 172, 171-179 (1999).

21. E. Betzig, J. K. Trautman, J. S. Weiner, T. D. Harris, and R. Wolfe, "Polarization contrast in near-field scanning optical microscopy," Appl. Opt. 31, 4563-4568 (1992).

22. K. Nakajima, Y. Mitsuoka, N. Chiba, H. Muramatsu, T. Ataka, K. Sato, and M. Fujihira, "Polarization effect in scanning near-field optic/atomic-force microscopy (SNOM/ AFM)," Ultramicroscopy 71, 257-262 (1998).

23. M. Born and E. Wolf, Principles of Optics (Cambridge Univ. Press, 1999).

24. K. G. Lee, H. W. Kihm, J. E. Kihm, W. J. Choi, H. Kim, C. Ropers, D. J. Park, Y. C. Yoon, S. B. Choi, D. H. Woo, K. Kim, B. Lee, Q. H. Park, C. Lienau, and D. S. Kim, "Vector field microscopic imaging of light," Nat. Phot. 1, 53-56 (2007).
25. S. Vignolini, F. Intonti, F. Riboli, D. S. Wiersma, L. Balet, L. H. Li, M. Francardi, A. Gerardino, A. Fiore, and M. Gurioli, "Polarization-sensitive near-field investigation of photonic crystal microcavities," Appl. Phys. Lett. 94, 163102 (2009).

26. E. Verhagen, M. Spasenović, A. Polman, and L. Kuipers, "Nanowire plasmon excitation by adiabatic mode transformation," Phys. Rev. Lett. 102, 203904 (2009).

27. M. Spasenović, D. van Oosten, E. Verhagen, and L. Kuipers, "Measurements of modal symmetry in subwavelength plasmonic slot waveguides," Appl. Phys. Lett. 95, 203109 (2009).

28. M. Burresi, R. J. P. Engelen, A. Opheij, D. van Oosten, D. Mori, T. Baba, and L. Kuipers, "Observation of polarization singularities at the nanoscale," Phys. Rev. Lett. 102 033902 (2009).

29. Y. Wu, M. Villanueva-Ibanez, C. L. Luyer, J. Shen, and J. Mugnier, "Application of multi-wavelength m-lines spectroscopy for optical analysis of sol-gel prepared waveguide thin films," Proc. SPIE 5946, 396-407 (2005).

30. R. Hillenbrand and F. Keilmann, "Complex optical constants on a subwavelength scale," Phys. Rev. Lett. 85, 3029-3032 (2000)

31. M. Sandtke and L. Kuipers, "Spatial distribution and nearfield coupling of surface plasmon polariton Bloch modes," Phys. Rev. B 77, 235439 (2008).

32. A. L. Campillo, J. W. P. Hsu, K. R. Parameswaran, and M. M. Fejer, "Direct imaging of multimode interference in a channel waveguide," Opt. Lett. 28, 399-401 (2003).

33. B. E. A. Saleh and M. C. Teich, Fundamentals of Photonics (Wiley, 1991). 